\title{
LA PATERNIDAD COMO EJERCICIO DE LA MASCULINIDAD IGUALITARIA
}

\section{FATHERHOOD AS AN EXERCISE OF EGALITARIAN MASCULINITY}

Tomás Israel López Marín.

Fundación Cepaim - España

ilopezmarin.ilm@gmail.com

Resumen: El objetivo de este artículo es entender como el ejercicio de la paternidad en base a la igualdad supone la oportunidad para la deconstrucción de la masculinidad hegemónica tradicional hacia un modelo de masculinidad igualitaria. Desde la propuesta de las masculinidades igualitarias, la paternidad se presenta como la oportunidad de reflexionar sobre la idea de masculinidad que venimos aprendiendo de manera tradicional, para poder desaprender los roles de género adquiridos durante toda la vida. Es por ello que la paternidad igualitaria supone un modelo de resistencia hacia las ideas tradicionales de lo que significa ser un hombre, potenciando nuevas masculinidades igualitarias.

Palabras clave: masculinidad, género, paternidad, igualdad, parentalidad, socialización.

\begin{abstract}
The objective of this article is to understand how to exercise fatherhood on the basis of equality supposes the opportunity for the deconstruction of the traditional hegemonic masculinity towards a model of egalitarian masculinity. From the proposal of egalitarian masculinities, fatherhood is presented as the opportunity to reflect on the idea of masculinity that we have been learning in a traditional way, in order to unlearn the gender roles acquired throughout life. That is why egalitarian fatherhood is a model of resistance towards traditional ideas of what it means to be a man, promoting new egalitarian masculinities.
\end{abstract}

Key Words: masculinity, gender, fatherhood, equality, parenting, socialization.

\section{Introducción}

Durante siglos, a lo largo de la historia de la humanidad, se ha venido construyendo la idea de la masculinidad hegemónica: ese hombre que todo lo puede, el hombre fuerte, protector y proveedor. Un modelo de masculinidad tradicional que, de manera inequívoca, genera serios problemas al no ajustarse a la realidad de un 
contexto cambiante y dinámico que, conduce a identidades insanas y frena el desarrollo de la igualdad. Algo que ha sido permeado en la paternidad. Una paternidad en muchas ocasiones distante y ausente, que ha servido de modelo de reproducción social hacia las nuevas generaciones.

Las nuevas masculinidades o masculinidades alternativas proponen replantear la idea de masculinidad y desaprender los roles de género adquiridos durante toda la vida y perpetuados a lo largo de siglos. La masculinidad, como construcción, es el conjunto de características que la sociedad supone que definen a los hombres, por ser propias de su sexo o al menos acentuadas en él. Usualmente abarca rasgos tanto biológicos como culturales. Mientras que, distintas corrientes académicas han señalado que la masculinidad es un conjunto de atributos, que los comportamientos y roles asociados con los hombres, niños y adultos, que son definidos social y biológicamente, distinta de la definición del sexo anatómico masculino. En esta línea de pensamiento académico, desde los estudios de género, se ha señalado que la masculinidad es también la construcción cultural de género que designa el rol de los varones en las sociedades. Desde esta perspectiva es desde donde pretendo abordar las disidencias dentro del modelo heteropatriarcal de crianza. Un modelo educativo que, aunque vigente, es profundamente obsoleto e injusto. Es necesario abordar la paternidad desde la perspectiva de los hombres igualitarios, en plena coherencia con la construcción de una sociedad igualitaria y equitativa.

\section{Desarrollo}

\subsection{Masculinidad tradicional hegemónica y paternidad}

Nos encontramos ante una crisis de la identidad masculina tradicional y con ella, el resurgir de nuevos modelos de masculinidades desde la perspectiva de género. Fruto de este largo proceso de aculturación, es necesario considerar que las consecuencias del machismo, y el de haber sido socializados en una sociedad de estructura heteropatriarcal, en los propios hombres, supone un largo proceso de contradicciones, tensión y malestar ante maneras disidentes de entender la masculinidad.

Ante este nuevo escenario, el reto de la conciliación corresponsable, la deconstrucción de la identidad masculina y el surgimiento de grupos de hombres igualitarios son vías necesarias para seguir construyendo el camino hacia la igualdad real entre hombres y mujeres. Vías cada vez más necesarias para construir un nuevo modelo de masculinidad alejada de los contravalores de la masculinidad hegemónica tradicional. Ante esta realidad, la paternidad y sus diferentes modelos deben de asumir el reto del compromiso en clara perspectiva de género con la finalidad de entender la responsabilidad que los padres tenemos para con las futuras generaciones. 
Entre los rasgos convencionalmente considerados masculinos, en tanto el conjunto de características al rol tradicional del varón, se incluyen la valentía, la independencia, y la asertividad. Sin embargo, las valoraciones de estas características y sus aplicaciones específicas varían según la ubicación y el contexto, dependiendo de factores sociales y culturales.

Otras fuentes también consideran la fuerza muscular e intelectual, la valentía, la virilidad, la ambición, el dominio de habilidades, el estoicismo, la agresividad, la competitividad, la seguridad, la auto-represión de la afectividad y el ejercicio de la violencia como rasgos de masculinidad. De este modo, a lo largo de la historia, los varones hemos experimentado, desde niños, una gran presión social para responder a las expectativas culturales de masculinidad, a través de comportamientos asociados a esos atributos. Así mismo en este contexto también ha provocado reacciones de hostilidad y represión cuando aparecen mujeres exhibiendo estas características.

Es por ello que necesitamos salir de la hipermasculinización que relaciona la masculinidad tradicional hegemónica con acciones como proteger, proveer o procrear, construyendo una historia propia entre varones que representa a la mujer como la "alteridad absoluta sin reciprocidad" (Sambade Baquerin, 2014). Por lo tanto, la transformación social feminista que debemos experimentar los hombres, desde la paternidad, debe erosionar los tres ejes de identificación-confirmación de la masculinidad patriarcal, algo que, por otro lado, ocasionaría el malestar de aquellos hombres, que, condicionados socialmente para desarrollar este modelo, lo veían ahora, impugnado y, con ello, frustradas sus pretensiones de reconocimiento social.

El eje de la masculinidad que hemos conocido en el pasado y que aún sigue siendo vigente, a día de hoy, puede caracterizarse como "virilidad" y pivota sobre una idea central: los hombres deben ser valientes, deben saber imponerse sobre su entorno.

Todo un ideario identitario que ejerce una presión sobre los niños para que adopten el género masculino prescrito que desde siempre ha sido siempre terriblemente fuerte. Perpetuando las relaciones sociales de poder, en relación a los sexos, se construyen y perpetúan culturalmente, por lo que, a través de una socialización igualitaria y la coeducación, podemos transformar estas ideas machistas y androcéntricas sobre las relaciones de género (Tellez, 2017).

La masculinidad tradicional hegemónica, se encuentra centrada en la "naturalización" de su hegemonía, donde cuando esta falla, y es cuestionado su poder, aparecen otras formas más explicitas de dominación (Bourdieu, 2005). Una estructura de poder desigual enmarcado en un modelo de masculinidad tradicional trasmitido a los hijos desde un modelo de paternidad ausente, que se va configurando sobre una lógica de poder desde la dimensión relacional.

La constante repetición y ejercicio de estas actividades se reviste, por lo tanto, de una cultura androcéntrica en la que se valoran como características positivas; fuerza, 
agresividad, competitividad, racionalidad, valentía y mucho más el protagonismo. Lo anterior logra posicionar la masculinidad como lo superior, lo importante, considerándolo además como algo natural y biológico. Por eso es común la expresión de que quien debe mandar en la familia, en la política y las finanzas es el hombre, el padre, porque es "natural" (Valera Bautista, 2020).

\subsection{Masculinidades igualitarias y Paternidades}

Debemos entender que la igualdad entre hombres y mujeres no será plena mientras que no transformemos el orden patriarcal que sigue sustentando una clara diferencian jerárquica entre sexos (Salazar, 2012). Y esto, conlleva de manera irrevocable, revertir la estructura de un sistema perverso, y profundamente desigual que, sigue manteniendo una concepción de lo humano basada en los privilegios masculinos y en la negación de la diversidad.

Esta situación de desigualdad y las relaciones de poder generadas, donde los hombres poseen claros privilegios sobre las mujeres, también se proyecta en las relaciones desarrolladas entre el padre con sus hijos e hijas, suponiendo un claro obstáculo en el desarrollo de una relación plena a nivel emocional, una relación capaz de poner los cuidados como eje de la misma.

La teoría feminista ha puesto de manifiesto que estas exigencias sociales emergen de la supremacía social y política de los hombres y no solo la reproducen, sino que también la legitiman, induciendo la creencia cultural de que los hombres tienen derecho a la misma. La concepción tradicional y hegemónica de "masculinidad" se vincula, y legitima la idea de que existe una relación natural entre el sexo masculino y la detención social del poder, especialmente en lo que refiere a la posición relativa de los hombres respecto de las mujeres. Esta ideología, conocida popularmente como machismo, ha condicionado a los hombres para desarrollar conductas caracterizando el riesgo, la ausencia de responsabilidad y el ejercicio de la violencia. Actualmente, la ideología machista es rechazada oficialmente en las sociedades contemporáneas. Sin embargo, en la práctica todavía existen desigualdades o presión por perpetuarla desde nuevos modelos postmachistas que velan por darle perpetuidad al heteropatriarcado.

El modelo de masculinidad tradicional hegemonía, ya no sirve. La denominada "cuarta ola feminista', que ve la luz definitivamente en las manifestaciones feministas del 8 de marzo de 2017 y 2018, es un reflejo de la necesidad de incorporar a los hombres ante el compromiso de la construcción de una sociedad igualitaria. Es evidente la necesidad de deconstruir la masculinidad hegemónica para profundizar en las identidades masculinas alternativas, disidentes, nuevas que nos permitan detenernos en la que más nos conciernen: las masculinidades igualitarias. Por eso, las nuevas masculinidades buscan una alternativa a ese modelo hegemónico que IQUAL. REVISTA DE GÉNERO E IGUALDAD, 2022, 5, 127-144 
incorpore la perspectiva de género. Un modelo de masculinidad capaz de mostrarnos nuevos, y diversos, modelos de paternidades, con perspectiva igualitaria.

En el siglo XXI está siendo el de la revolución del padre, la de esos hombres que han transformado su rol paterno, que están siendo padres de una forma tanto cuantitativa como cualitativamente muy diferente al ejemplo que tuvieron como hijos. Ha cambiado la sociedad y, sobre todo, el papel de las mujeres, empoderadas e incorporadas masivamente al ámbito laboral, lo que ha promovido la necesidad de una mayor implicación de los hombrees en los cuidados (Bacete, 2021).

La apuesta por el cuidado es un factor indispensable para la construcción de una sociedad más igualitaria. Implicarnos como hombres en el cuidado es, ir mucho más allá de aspectos como la paternidad. Es construir la masculinidad desde las relaciones en equilibrio y vivir la crianza de nuestros hijos e hijas, y el cuidado como espacios propios. No como terreno en el que los hombres somos meros invitados o en el que las mujeres nos indican y nos dicen cómo hay que hacer las cosas, sino que todos y todas somos ciudadanos de pleno derecho dentro del hogar y en la implicación con otras personas. Este es el verdadero reto de los padres igualitarios ante el desafío de la igualdad de género. Este es el verdadero desafío de los hombres igualitarios.

\subsection{Paternidades ausentes / Paternidades presentes}

Tal y como expone Subirats, mientras que el género femenino ha ido evolucionando muy rápidamente en los lugares en que esta evolución no ha sido duramente reprimida, el género masculino está evolucionando de forma mucho más lenta, y existen unas mayores resistencias al cambio, que se hacen patentes de diversa manera en las distintas culturas y países, pero que, bajo formas variables, tienden a manifestarse en todo el mundo (Subirats Martori, 2020). Por tanto, no puede sorprendernos que sean las mujeres quienes han iniciado una renovación del género femenino.

La ruptura del equilibrio publico/privado plantea el que es uno de los grandes obstáculos para la igualdad del siglo XXI, la denominada conciliación entre la vida profesional y la personal/familiar (Salazar, 2018). Un concepto, el de la conciliación, que de manera mayoritaria se está entendiendo perversamente como una obligación de las mujeres, pero no de los hombres. Una realidad que exige, de manera innegable que las mujeres se conviertan en la práctica en personas explotadas que deben acumular una doble jornada laboral, en el mejor de los casos, sumando al trabajo fuera de casa, el trabajo doméstico.

El reto que debemos asumir, como hombres, es el de asumir que todo lo que se desarrolla en el ámbito privado, desde el cuidado de las hijas y los hijos al mantenimiento del hogar, debe ser una responsabilidad compartida. Por eso, 
deberíamos hablar más de "corresponsabilidad" que de conciliación. La corresponsabilidad implica asumir que todos estos trabajos, no les corresponden "naturalmente" a ellas, sino que también debe formar parte de nuestra agenda como hombres, y como padres.

Asumir el cuidado como parte indispensable de nuestra vida, es darnos la oportunidad de desarrollar una serie de capacidades, habilidades y emociones que se traducirían en nuestra manera de entender e interactuar tanto con el espacio público, como con el espacio privado. Todas estas herramientas, que desde el modelo heteropatriarcal se ha vinculado como "características femeninas", pasarían a formar parte de nuestra manera de desenvolvernos también en el trabajo, en nuestras relaciones con nuestros iguales.

Se trataría, por tanto, de incorporar a nuestras vidas los principios y los valores que, desde el feminismo se ha identificado con la ética del cuidado. El cuidado como eje indiscutible de la vida pública y privada, nos aportaría, sin lugar a duda, en una mayor capacidad para poder ponernos en el lugar de otras personas, así como de un modo más constructivo en la resolución de los conflictos que de la convivencia se generan.

Los padres, por ausencia o presencia, aportan modelos identitarios de referencia sobre qué es ser un hombre que producirán efectos profundos y que perdurarán toda la vida, sobre todo en la construcción de las identidades y expectativas vitales tanto de sus hijas como de sus hijos (Bacete, 2017).

Ante la dualidad del padre ausente / padre presente, desde la perspectiva de los hombres igualitarios, debemos de entender cómo, para los hombres es un gran reto ser padres hoy, estar dispuestos a ser padres "no patriarcales", cuya autoridad no sea mayor que la de la madre y que sea capaz de compartir labores domésticas, crianza y formación de la familia. Ser una persona con la seguridad suficiente para educar con afecto, proteger sin dominar y saber cuidar para, convertirse en un padre coresponsable. Tal y como expone Bacete (2017) cada vez contamos con mayor evidencia científica de que la implicación de los hombres en la crianza y los trabajos reproductivos son un factor clave para la transformación de la realidad hacia paradigmas sociales y de relación más justos e igualitarios.

\subsection{Paternidad, afecto y cariño}

Nos encontramos un momento en el que la identidad masculina y la paternidad se nutren de dos modelos del ser padre. Uno cifrado en los rasgos tradicionales, y otro que va surgiendo con referentes y características nuevos que proyectan un ejercicio de la paternidad basado en el respeto, el cariño y el afecto. 
Entender la paternidad como un espacio para el crecimiento emocional, es sin duda, la oportunidad para avanzar en un modelo de masculinidad disidente del modelo heteropatriarcal. Una oportunidad para encontrarnos con nosotros mismos en el plano afectivo.

Cuando somos padres comprometidos, hay un momento inevitable de crisis. Dejamos de vivir alrededor de nuestro ombligo y nos convertimos en seres cuidadosos en vez de ser hombre de cuidado. Ya no somos el centro de la existencia. Otro ingrediente de la crisis es que la paternidad consciente nos hace enfrentarnos a un mundo emocional inmenso para el que a veces, nos faltan códigos, referencias y herramientas (Bacete, 2017).

Tal y como afirma Salazar (2012), la igualdad entre hombres y mujeres no será plena mientras que no transformemos el orden patriarcal que sigue sustentando una clara diferencian jerárquica entre sexos. Un sistema perverso, y profundamente desigual que, sigue manteniendo una concepción de lo humano basada en los privilegios masculinos y en la negación de la diversidad. Esta negación de la diversidad también incluye ese rechazo a cualquier otro modelo de masculinidad más allá de los márgenes de la masculinidad tradicional hegemónica.

Es necesaria la construcción de espacios para repensar que significa la masculinidad en el Siglo XXI, en un contexto tan cambiante y dinámico, donde la división de sexual de las tareas, o el reparto de roles por cuestión de genero sigue provocando una profunda desigualdad de carácter histórica. Un reto de profundas raíces que cada vez más nos invita a la ruptura de un sistema patriarcal (Sanfelix, 2020).

El cambio cultural que vive la humanidad en los últimos años está superando las condiciones sociales en el campo de las relaciones entre los géneros, no puede ponerse en cuestión la capacidad reflexiva femenina para crear relaciones más igualitarias y más placenteras.

La paternidad es una de las formas sociales mediante las cuales el hombre manifiesta su identidad y poder masculino. Esta etapa de la masculinidad, se expresa de múltiples y variadas formas en función de las expresiones culturales, que responden y manifiestan estereotipos y roles sociales proyectados como prácticas culturales únicas (Montesinos, 2002). En este sentido, los cambios producidos a nivel económico, político, social y cultural en la sociedad occidental, evidencian la necesidad de construir nuevos modelos de ser hombres, y, sobre todo, nuevos padres que reconozcan a hombres y mujeres como sus iguales, asumiendo responsabilidades domésticas, familiares y laborales.

De este modo, Montesinos (2002) afirma:

La nueva paternidad, es entendida como aquella expresión de la masculinidad emergente que representa la capacidad crítica a los modelos tradicionales de IQUAL. REVISTA DE GÉNERO E IGUALDAD, 2022, 5, 127-144 ISSN. 2603-851X DOI. http://dx.doi.org/10.6018/iqual.490701 
género, y cuya esencia permite concentrar el poder de la figura masculina. Se trata, en consecuencia, de asociar una nueva forma de ejercer el poder y de representar la autoridad, social y familiar, a la figura de la nueva masculinidad-nueva paternidad. (p. 198)

Las áreas de intervención en el ámbito social, educativo y sanitario son de especial interés para hombres y mujeres, así como asociaciones de hombres que quieren promover en la actualidad nuevos modelos de paternidad, nuevos padres participativos en la relación doméstica, laboral y familiar, y padres igualitarios en el reconocimiento de la igualdad y equidad de hombres y mujeres. Para ello, es necesario promover nuevos modelos sociales que fomenten la participación de hombres, y, sobre todo, de padres, promoviendo leyes que favorezcan la conciliación de la vida familiar y laboral, y estableciendo nuevas propuestas de actuación a partir de los modelos sociales de masculinidad y paternidad tradicionales.

Para quienes tenemos el interés de lograr la igualdad de género, como derecho humano aún para quienes no le dan tanta importancia, resulta interesante entender que el cambio de una paternidad autoritaria y poco afectiva como la de antaño a otra más afectiva, cercana y participativa es deseable; porque es un indicador de igualdad y desarrollo y de un cambio social deseable por incluyente y más equitativo (Valera Bautista, 2020).

\subsection{Paternidades y coeducación}

La forma en que educamos a nuestras hijas y a nuestros hijos, desde el modelo patriarcal hegemónico, les hace un flaco favor. Reprimimos la humanidad de los niños. Definimos la masculinidad de una forma muy estrecha. La masculinidad es una jaula muy pequeña y dura en la que metemos a los niños. Enseñamos a los niños a tener miedo al miedo, a la debilidad y a la vulnerabilidad. Les enseñamos a ocultar quienes son realmente porque tienen que ser [...] hombres duros. (Ngozi Adiche, 2015).

El papel de la educación y de la crianza responsable, para el desarrollo de una sociedad igualitaria y comprometida para le erradicación de las desigualdades por motivo de sexo o género, es esencial. Por ello, el debate sobre la reflexión teórica y práctica de un nuevo modelo educativo, inclusivo, y respetuoso, debe responder a las características de una sociedad que parece avanzar más rápido que las instituciones educativas.

Es una realidad que los niños y las niñas acceden al sistema educativo reglado, tras haber interiorizado un esquema social relativo al rol de género. Unos esquemas que se irán reproduciendo conforme avance su proceso educativo. Motivo por el cual, las escuelas, como pequeños microcosmos situados en la realidad social que los rodea 
supone un claro núcleo de construcción y refuerzo de la masculinidad tradicional hegemónica (Rodríguez Menéndez, 2007).

También es necesario reflexionar sobre la democratización de las relaciones de género. Los roles de género se encuentran tan profundamente enraizados que a menudo los seguimos reproduciendo incluso cuando chocan de manera frontal con nuestros verdaderos deseos, con nuestras necesidades, y con nuestra felicidad. Los roles de género son difíciles de desaprender, $y$, por tanto, es importante intentar que nuestros hijos e hijas los rechacen desde el principio (Ngozi Adiche, 2017).

Los hombres con prácticas más igualitarias y cuidadoras en sus vidas son también más sanos y felices. Son capaces de mostrar una realidad plural y diversa a sus hijos e hijas, a ser críticos con el entorno.

Enseñar a nuestras hijas e hijos a cuestionar el lenguaje. El lenguaje como depositario de nuestros prejuicios, creencias y presunciones. Pero para enseñárselo, primero deberíamos cuestionar nuestro propio lenguaje (Ngozi Adiche, 2017).

Cuando los hombres empiezan a participar en los cuidados, a compartir los trabajos reproductivos, las relaciones de pareja mejoran y son más satisfactorias. $Y$, sobre todo, se expanden las posibilidades de las hijas e hijos de las parejas más igualitarias, que tienen, que tienen mejores resultados académicos o enferman menos, y eso es lo que los hombres deseamos como padres (Bacete, 2017).

Por tanto, es necesario poner en valor la importancia que tiene la implicación positiva de los padres en la crianza de sus hijos y sus hijas como un elemento esencial para generar relaciones basadas en el apego seguro en el ámbito familiar.

La corresponsabilidad paternal es punto clave en el proceso de igualdad de género porque permite desde la familia impulsar cambios que se reflejan en el ámbito social, político y laboral, que darían oportunidades iguales a mujeres y hombres, por tal motivo es importante considerar que para propiciar que las paternidades corresponsables cobren auge es necesario fomentar acciones desde los gobiernos como: generar políticas de igualdad para promover una cultura institucional que sensibilice al personal sobre el hecho de que cuidar y educar es una tarea de mujeres y hombres (Valera Bautista, 2020).

\section{Análisis}

Actualmente, las iniciativas públicas que empezaban a considerar a los hombres como aliados necesarios en el camino hacia la igualdad, así como sujetos de intervención desde una perspectiva feminista: el teléfono para hombres o el compromiso de ampliar los permisos de paternidad. Desde esta perspectiva, la igualdad real entre hombres y mujeres requiere de un nuevo planteamiento en el ámbito público y en el ámbito privado. Un nuevo sistema social basado en la igualdad 
supone claros beneficios para hombres y mujeres, pero eso requiere de una nueva organización social donde el hombre renuncia a sus privilegios y se implique activamente en el cambio, al tiempo que sean partícipes de la mejora de la vida de las mujeres.

Puede parecer que el orden de factores no altera el producto, pero en este caso lo hace, al menos subjetivamente, porque no es lo mismo pedir a los hombres solidaridad que colaboración, ayuda que corresponsabilidad, apoyo que implicación en el diseño y construcción de un futuro compartido en el que mujeres y hombres tengamos los mismos derechos, las mismas oportunidades, y las mismas responsabilidades. Reconocer que la igualdad necesita promover el cambio de los hombres sin dejar por ello de destacar que la mejora de su bienestar también es un objetivo legítimo de las políticas de igualdad en áreas como la paternidad, las relaciones laborales, la educación, la violencia machista o la vida afectiva y familiar es el resultado de un proceso que se viene produciendo como consecuencia de una combinación de factores:

La conciencia del movimiento feminista de que tras conquistar la igualdad legal ve necesaria la participación e implicación de los hombres para conseguir la igualdad real, el trabajo de los hombres por la igualdad que llevan décadas criticando los modelos masculinos tradicionales hegemónicos e intentando implicar a los hombres frente al machismo, las múltiples Conferencias sobre la Mujer y de las Naciones Unidas que recomiendan alentar la corresponsabilidad de los hombres en la promoción de la igualdad, y los buenos resultados cosechados por las experiencias locales (Jerez, Euskadi,..) "para promover la concienciación, participación e implicación de los hombres a favor de la igualdad de sexos", tal y como aporta la iniciativa Gizonduz, una iniciativa pionera del gobierno vasco, impulsada por Emakunde-Instituto Vasco de la Mujer-, dirigida a promover la concienciación, participación e implicación de los hombres en pro de la igualdad de mujeres y hombres

El retraso en incorporar de manera activa a los hombres como beneficiarios directos de las políticas de igualdad ha tenido consecuencias indeseadas, ayuda a explicar la existencia de un sector creciente de la población que tiene la percepción subjetiva, y errónea, de que algunas leyes (sobre todo relativas a violencia de género y la custodia de la prole en las separaciones) van dirigidas contra los hombres y por tanto contra la igualdad.

Debemos evitar el error de que las urgencias defensivas nos impidan analizar y superar algunos de los errores del pasado que han contribuido al desencanto y el rechazo a las políticas de igualdad. Superar las dudas sobre la necesidad de contar con los hombres para defender las conquistas en peligro y recuperar la iniciativa en el impulso de la igualdad entre hombres y mujeres, sin olvidar que la prioridad sigue siendo promover el empoderamiento de las mujeres y la lucha contra las IQUAL. REVISTA DE GÉNERO E IGUALDAD, 2022, 5, 127-144 ISSN. 2603-851X DOI. http://dx.doi.org/10.6018/iqual.490701 
desigualdades persistentes, implica asumir que es un error dejar en segundo lugar el cambio de los hombres como sujetos de intervención desde una perspectiva de género.

Un apoyo que siempre ha contado con aquellos sectores del movimiento feminista que ven la igualdad como una aspiración democrática inalcanzable sin aliarse con el movimiento de hombres por la igualdad, que (todo hay que decirlo) nunca han puesto en peligro los recursos destinados a las mujeres que tanto les ha costado conseguir. Que las prioridades sean la erradicación de las violencias contra las mujeres y la implicación de los hombres en lo doméstico (paternidad, cuidados,...), no impide que haya áreas como el fracaso escolar, la exclusión social o la salud, en las que la situación de los hombres parece ser peor que la de las mujeres, como clara consecuencia derivada de un modelo de masculinidad hegemónica tradicional, que falten estudios sobre los hombres o el interés que tiene ayudar y apoyar a los hombres a que elijan profesiones no tradicionales y estudios de humanidades.

La experiencia de Noruega, con la mayor igualdad entre los sexos del mundo y el segundo lugar entre los países de Europa en asesinatos machistas, nos recuerda que la igualdad para ser efectiva necesita acabar, al mismo tiempo, con las desigualdades estructurales que padecen las mujeres y con el machismo; que la sociedad igualitaria que propone el feminismo no será posible sin el cambio y la implicación de los hombres, como agentes activos de la transformación.

En los años siguientes la igualdad llegó a ser el discurso social hegemónico, hasta el punto de que costaba encontrar quien se opusiera públicamente y los medios de comunicación tenían que buscar personajes esperpénticos para defender el machismo en los debates. Hoy la situación es distinta, el discurso postmachista ha conseguido que amplios sectores de la población crean que la igualdad no beneficia a la mayoría, sino que busca privilegios para las mujeres. Un claro síntoma de la repercusión mediática de ciertas tendencias políticas en boga en la España actual, y que cristaliza en la sociedad. Algo similar, sucede actualmente con los movimientos que hace referencia a gestos como la culpabilidad maternal y o el movimiento madres arrepentidas.

No hay duda de que ellos han renovado su discurso y los hombres por la igualdad no hemos llegado a la mayoría de los hombres para convencerlos de las virtudes del cambio, pero no es menos cierto que las políticas públicas de igualdad se han olvidado de los hombres como sujetos de intervención con perspectiva de género y este olvido ha facilitado que los postmachistas usen el disfraz de defensores de la igualdad efectiva. Las iniciativas institucionales para ayudar a los hombres en el cambio han sido locales y/o anecdóticas.

Ninguna fuerza política apuesta por incorporar a los hombres como beneficiarios de las políticas de igualdad, a sus iniciativas les ha faltado el respaldo necesario y sus 
militantes consideran la igualdad un tema de mujeres, que ellas protegen como un coto privado y a ellos les resulta más cómodo acotar desde sus responsabilidades partidarias o institucionales. La falta de sensibilidad ante fenómenos como el fracaso escolar de los chicos, el precio que pagan niños y hombres por ir de machos por la vida, la importancia de ampliar los permisos de paternidad o la necesidad de dotar de prestigio social lo doméstico y los cuidados, son anécdotas que sumar.

Defender las conquistas amenazadas y luchar por una salida de la crisis que incremente el empoderamiento de las mujeres fomentando la corresponsabilidad, necesita una mayoría social que incorpore a los hombres. A estos se les puede y se les debe exigir que renuncien a sus privilegios sin contraprestaciones, porque es justo y necesario, pero si queremos incorporarlos activamente al cambio hay que lograr que se sientan parte del mismo.

La igualdad y los hombres parecen como el agua y el aceite, irreconciliables pero imprescindibles en la dieta mediterránea. Los hombres, los grandes beneficiarios del Patriarcado, son imprescindibles para el cambio; su implicación dependerá también de que además de justa, vean que la igualdad les beneficia y no les compensa el precio que pagan por los privilegios.

\section{Conclusiones}

En los últimos años, el discurso público en torno a la paternidad ha experimentado cambios importantes. La distribución tradicional de roles entre mujeres y hombres excluyó al padre de la participación directa en la prestación de cuidados, pero en las últimas décadas, las familias en las sociedades occidentales han experimentado enormes transformaciones que han remodelado estos roles. El ingreso de las mujeres al mercado laboral, la tendencia hacia relaciones más igualitarias, junto con otros cambios demográficos y familiares, es decir, baja fecundidad, aumento de las tasas de divorcio o convivencia, identificados como la segunda transición demográfica, han llevado a una redefinición de las responsabilidades del cuidado, en particular en lo que respecta al cuidado infantil. En consecuencia, los padres han asumido roles más destacados en esta área. En este contexto, analizar el discurso social de los padres respecto a sus hijos e hijas, y las implicaciones que este cambio tiene en la crianza resulta una cuestión de relevancia pública, política y social.

Las actitudes paternales tradicionales no han desaparecido por completo, pero se percibe una mayor implicación de los padres en las relaciones padre-hijo. Han aparecido nuevos modelos de "padres" alternativos al tradicional "sostén de la familia", que han dado lugar a relaciones basadas en el amor y no solo en el poder (Bonino, 2003). 
Si bien las políticas familiares en el contexto europeo han promovido un modelo familiar igualitario y existe un cierto consenso generalizado de que este tiene prioridad dentro de las políticas sociales nacionales, las políticas familiares españolas han sido descritas como recientes, inconsistentes e inciertas en el modelo familiar que tienen como objetivo promover. Por tanto, estos países representan ejemplos estratégicos para analizar la influencia que tienen las dimensiones estructurales en el ejercicio de la paternidad.

La reciente transformación de la paternidad ha recibido una atención considerable en los círculos académicos. Sin embargo, el auge del movimiento feminista durante la década de 1970 trajo consigo una nueva perspectiva sobre la paternidad (Vuori, 2009). Desde entonces, la paternidad se ha convertido en un objeto de creciente interés académico, ya que se ha conceptualizado como un mecanismo fundamental para lograr la igualdad de género. Hemos entendido la "paternidad involucrada" como una noción que representa una transformación del rol tradicional de sostén de la familia y que promueve la crianza, el cuidado de los niños y la crianza compartida.

Estos padres involucrados dedican más tiempo al ejercicio de la paternidad y realizan actitudes más igualitarias en sus discursos, aunque se mantienen ciertos valores, tradicionalmente asociados a la masculinidad. presente en sus manifestaciones y comportamientos. A pesar de la transformación paulatina, estos nuevos padres continúan desempeñándose como sustentadores primarios y cuidadores secundarios, ya que se han socializado con valores contradictorios en cuanto a su rol de padres.

De alguna forma, podemos vincular los cambios a las estructuras sociales, las políticas familiares y las expectativas de género hacia la paternidad, tres dimensiones que conectan a la sociedad, las instituciones y los individuos. En consecuencia, los diferentes modelos de paternidad son el resultado de la convergencia de las macroestructuras con microelementos y comportamientos concretos del individuo, ya que "las prácticas de paternidad son vistas como resultado de los diversos procesos de negociación dentro de estas diferentes estructuras". Estas estructuras conforman, al igual que la estructura de género definida por Risman (2017), "un sistema dinámico: cuando una parte cambia, puede desencadenar una reacción en cadena como un juego de dominó. Existe una causalidad dinámica recursiva entre los yoes individuales, las expectativas de interacción, la ideología cultural y la estructura organizativa" ( $p$. 210).

En ese sentido, las diferentes estructuras e instituciones están interconectadas e influyen entre sí en la socialización de los ciudadanos -el proceso de interiorización de los valores, normas y expectativas de la sociedad-y los procesos cognitivos desarrollados en etapas posteriores, durante la vida, los ciclos de los individuos, cuando las expectativas y los incentivos sociales relacionados con las categorías 
sociales de los individuos, como el género, remodelan o refuerzan los procesos de aprendizaje ocurrieron durante la infancia.

En consecuencia, el alcance estructural de la paternidad debe entenderse como una interacción compleja de diferentes dimensiones, externas e internas de los agentes, que ejercen su fuerza directa o indirectamente, a través de múltiples canales y procesos diversos. En consecuencia, como indica Browne (2013, p.153),

la política eficaz debe basarse en una imagen matizada no solo de cómo los padres están limitados por consideraciones económicas o impulsados por aspiraciones individuales, sino también de cómo están limitados por una compleja gama de factores estructurales. y factores institucionales.

Desde esta óptica, las limitaciones estructurales también influyen en las interacciones, ya que las tres estructuras sociales mencionadas anteriormente actúan como facilitadoras o barreras que generan hábitos y rituales para la participación de los padres, y también establecen las negociaciones con los padres, otros agentes, fundamentalmente con las madres. Los roles asumidos hacia la crianza representan la convergencia de las interacciones y negociaciones entre los diferentes agentes, la restricción de las estructuras externas y la internalización de las expectativas del otro generalizado (Browne, 2013).

Como narrativa de género, la paternidad involucrada funciona de múltiples maneras, ya que se entrelaza con diferentes repertorios de género. Cuando se cruza con la narrativa de la igualdad de género, la narrativa del género de la paternidad involucrada neutraliza la paternidad en formas que democratizan la organización de la vida familiar. Por el contrario, podemos encontrar casos en los que también se deconstruye, en muchos sentidos, la primacía de la maternidad, la narrativa de la paternidad involucrada se utiliza simultáneamente para preservar la noción de virilidad. Al enfatizar los atributos del cuidador masculino, podemos establecer que la paternidad es fundamentalmente diferente de la maternidad, lo que puede interpretarse como una forma de masculinizar las prácticas de cuidado y, por lo tanto, de (re) establecer una posición suficientemente masculina para sí mismo, que puede no distar de la igualdad entre hombre y mujer, manteniendo los roles propios.

En consecuencia, las restricciones estructurales generan disrupciones intergeneracionales que permiten generar nuevas ideas, nuevos campos de trabajo y nuevas limitaciones internas, características personales y dinámicas de grupo que influyen en la configuración del sentido compartido por el que entendemos la masculinidad, y la paternidad.

\section{Valoración}


Cada historia cuenta con una nueva posición masculina dentro de la familia, ya que son ellos los que deben adaptarse a las necesidades de su pareja, de la crianza y ajustar sus propias ambiciones laborales y responsabilidades domésticas para adaptarse a los requisitos laborales de ella, $y$, por ende, de la unidad familiar. Su énfasis en la elección, la paternidad y la igualdad de género muestra, sin embargo, que construir puestos de amas de casa inteligibles requiere mucho trabajo cultural. Admitir haberse ajustado a las demandas de una esposa es un desafío, ya que el cumplimiento amenaza su inteligibilidad como hombres. Cabe destacar que, a pesar de su énfasis en la elección, también juegan con la noción de sacrificio al mostrarse como facilitadores de las carreras de las mujeres. Si bien afirman ser socios flexibles y comprensivos que se invierten en el proyecto de "hacer que funcione", también logran (re) instalarse simultáneamente como en control al indicar cómo la orientación profesional de su pareja solo es posible debido a su voluntad de adaptar.

En este sentido existente en las historias, los aspectos sacrificiales son algo minimizados. Esto puede entenderse porque la idea de sacrificio no funciona particularmente bien con la narrativa cultural del voluntarismo y el amor mutuo entre individuos iguales y autónomos (como, por ejemplo, esbozado por Giddens 1992). Como también señala Swidler, una "persona que sabe lo que quiere y actúa en base a ese conocimiento no puede sacrificarse, ya que cualquier cosa que realmente quiera hacer no es un sacrificio". Como la narrativa de la elección en este sentido funciona como una estrategia para reparar su posición restaurando yoes inteligibles, también indica hasta qué punto el rol con connotaciones femeninas como "la base de apoyo" necesita ser restaurado para parecer suficientemente autoritario para un hombre.

Simultáneamente, sin embargo, la narrativa de la paternidad involucrada reconstruye aún más lo que podría parecer una elección de sacrificio en una oportunidad tanto significativa como deseable (para un hombre). A través de las historias de puericultura intensiva, la falta de carrera se convierte en ganancia de intimidad. Para estos hombres, estas nuevas masculinidades suponen la relación cercana con los niños obtenida a través del cuidado diario que parece ser la forma lógica de validar su orientación hacia el hogar, lo que subraya cómo el centrarse en el niño es un ideal normativo fácilmente accesible en el contexto concreto.

Sin embargo, no se puede decir que la narrativa de la paternidad involucrada, de la misma manera que la elección, restaure un yo masculino convencional. Más bien, esta historia expande la idea de lo que significa ser un hombre demostrando cómo se han agregado nuevas dimensiones de la emocionalidad y la orientación al cuidado a los recursos discursivos que brindan inteligibilidad masculina.

A pesar de activar lo que podría verse como una ideología "masculinista" para dar sentido a sus acciones, debe considerarse como una transgresión de las lógicas heteronormativas. Como sus estrategias discursivas pueden verse como cómplices de la idea hegemónica de la diferencia de género y como resistencia al orden de género 
tradicional, la ambivalencia y la contradicción entran en la forma en que se construye a sí mismo como "un tipo diferente de hombre" en lo que considera una "relación no convencional". "

En este sentido, estas estrategias, se basan en los valores hegemónicos de autonomía e independencia, y lo que se celebra es el "coraje, la fuerza y la determinación de estos hombres como hombres para participar en estas actividades potencialmente degradantes. Por esta razón, reclamar la no convencionalidad es quizás la forma más fácil de demostrar autonomía y, por lo tanto, masculinidad. Si bien a los hombres les parece significativo no presentarse como subordinados, como tal no expresan la autonomía de una manera masculinista. Más bien, sus historias reflejan lo que podríamos entender como una autonomía relacional. Aunque hay un "yo" que elige, estos hombres también suelen trabajar narrativamente con el sujeto plural de "nosotros". Este "nosotros" elige, piensa, le gusta, quiere y, lo que es más importante, es el "nosotros" el que organiza la vida familiar, indicando cómo se experimenta la vida cotidiana como una empresa conjunta y reforzando las historias generales de asociación y reciprocidad.

Esta reconstrucción es importante para los yoes relacionales producidos por estos hombres, ya que las divisiones asimétricas del trabajo en sus historias no solo desafían sus autoprestaciones masculinas, sino que también ponen en peligro los ideales de igualdad de género. En muchos sentidos, la narrativa de la cooperación reelabora las imágenes de sus relaciones para que se ajusten a la narrativa cultural de las relaciones democráticas.

Mientras reconstruyen su autodeterminación a través de la narrativa de la elección, estos hombres en general construyen lo que yo considero masculinidades no dominantes y orientadas a la igualdad de género o bien, nuevas masculinidades adaptadas a relaciones igualitarias. Siempre que se desempeñen con suficiente autoridad, la trama de los acuerdos laborales contractuales parece proporcionar a los hombres un marco contextualmente viable para posicionarse como hombres "nuevos" que brindan a su esposa (y su carrera) un amplio apoyo al asumir responsabilidades familiares. Vale la pena tener en cuenta, sin embargo, que, como logro situacional, la posición de "marido solidario" es concebible más fácilmente disponible y menos problemática, en el contexto de este proyecto de investigación y en una conversación con una investigadora más joven con una posición similar (alta) de clase media. No obstante, la ambivalencia con la que se construyen estas identidades incluso en esta situación demuestra cuán dominante es el miedo a parecer subordinado y cuán preocupante es el cumplimiento para la construcción de una masculinidad inteligible, incluso dentro del contexto de un régimen nórdico de igualdad de género.

Finalmente, considero que sostener que los hombres realizan masculinidades no dominantes y orientadas a la igualdad de género, la ambivalencia de estas representaciones identitarias resalta la necesidad de entender la construcción de la IQUAL. REVISTA DE GÉNERO E IGUALDAD, 2022, 5, 127-144 
identidad masculina como compleja e inconsistente. Si bien a menudo se destaca una igualdad de género donde los hombres ahora están muy involucrados en las tareas del hogar y el cuidado (especialmente en lo que respecta a las "cuotas paternales"), este artículo subraya que las transformaciones de género no se pueden entender como un proceso lineal. Aunque ciertamente es factible dentro del contexto de la clase media (alta) blanca y urbana, el logro del "nuevo" hombre debe entenderse como involucrado tanto con la complicidad como con la resistencia hacia las ideas tradicionales de lo que significa ser un hombre, potenciando nuevas masculinidades igualitarias.

\section{Bibliografía}

Bacete, R. (2017). Nuevos hombres buenos. La masculinidad en la era del feminismo, Barcelona, España: Península.

Bacete, R. y Solano, J. (2021). Papá. Barcelon, España: Planeta.

Bonino, L. (2003). Las nuevas paternidades. Cuadernos de Trabajo Social, 16(1), 171182.

Bourdieu, P. (2005). La dominación masculina, Barcelona, España: Anagrama.

Browne-Sartori, R. \& Castillo-Hinojosa, A. M. (2013). Análisis Crítico del Discurso de la representación intercultural en la prensa chilena. Convergencia, 20(62), 13-43.

Montesinos, R. (2002). Las rutas de la masculinidad. Ensayos sobre el cambio cultural y el mundo moderno, Barcelona, España: Gedisa.

Ngozi Adiche; C. (2015). Todos deberíamos ser feministas, Barcelona, España: Penguin Random House.

Ngozi Adiche; C. (2017). Querida ljeawele. Como educar en el feminismo, Barcelona, España: Penguin Random.

Risman, B. J. (2017). 2016 southern sociological society presidential address: Are millennials cracking the gender structure?. Social Currents, 4(3), 208-227.

Salazar Benítez; O. (2012). Otras masculinidades posibles. Hacia una masculinidad diferente y diferenciada. Recerca, 6, 87-112.

Salazar Benítez; O. (2018). El hombre que no deberíamos ser, Barcelona, España: Planeta.

Sambade Baquerín, I. (2014). Sobre las contradicciones de la razón moderna y la constitución de la subjetividad masculina. Prisma Social, 13, 2014, 787-851.

Sanfélix, J. (2020). La brújula rota de la masculinidad: Espacios de posibilidad para repensar la masculinidad en el Siglo XXI, 167-183, Valencia, España: Tirant Humanidades. 
Subirats Martori, Marina (2020). El género masculino, entre la obsolescencia y la impostación. En A. Téllez Infantes, J. E. Martínez Guirao y J. Sanfélix Albelda (Eds.), Hombres, género y patriarcado: Reflexiones, cuerpos y representaciones, Madrid, España: Dykinson, 19-34.

Téllez Infantes, A. y Verdú Delgado, A. (2011). El significado de la masculinidad para el análisis social. Revista Nuevas Tendencias en Antropología, 2, 80-103.

Téllez Infantes, A. (2017). Sobre cómo el machismo perjudica a los hombres. En A. Téllez Infantes, (Ed.) Igualdad de género e identidad masculina. Elche, España: Editorial electrónica de la Universidad Miguel Hernández, 6-20.

Téllez Infantes, A; Martínez Guirao, J. (2019). Hombres igualitarios, igualdad de género y reacciones masculinistas frente a la cuarta ola feminista. En A. Téllez Infantes, J. Martínez Guirao y J. Sanfélix Albelda, (Eds.) Masculinidades igualitarias y alternativas. Procesos, avances y reacciones, Valencia, España: Tirant Lo Blanch, 50-78.

Valera Bautista; G. (2020). Paternidades corresponsables e igualdad entre hombre y mujeres. Revista gestión de las personas y tecnologías, 37.

Vuori, J. (2009). Men's choices and masculine duties: Fathers in expert discussions. Men and masculinities, 12(1), 45-72.

Cómo referenciar este artículo/How to reference this article:

Tomás Israel López Marín, T.I. (2022). La paternidad como ejercicio de la masculinidad igualitaria. iQUAL. Revista de Género e Igualdad, 5, 127-144, doi: 10.6018/iqual.490701

Tomás Israel López Marín, T.I. (2022). La paternidad como ejercicio de la masculinidad igualitaria. [Fatherhood as an exercise of egalitarian masculinity]. iQUAL. Revista de Género e Igualdad, 5, 127-144, doi: $10.6018 /$ iqual.490701 